\title{
FOA Based Diagnosis Model for Multivariate Production Process
}

\author{
Yang Mingshun, Kong Xiangjian, Gao Xinqin, Liuyong and Li Yan \\ Faculty of Mechanical and Precision Instrument Engineering, Xi'an University of \\ Technology, Xi'an 710048, China \\ Yangmingshun@xaut.edu.cn,xiangjiankong0224@163.com,gaoxinqin@xaut.edu.cn, \\ liuyong@xaut.edu.cn,jyxy-ly@xaut.edu.cn
}

\begin{abstract}
Fault diagnosis for quality control during the multivariate production process is widely used to detect abnormal fluctuations, find out failure reasons and take measures to maintain the stability of the production system accordingly. The neural network methodology has become a main method in the field of intelligent diagnosis recently. However, it has certain deficiencies such as longer training time, slower convergence rate and easier falling into a local optimal solution easily. As a result, the effect of fault diagnose is influenced. Thus, this article proposes the idea of using the Fruit Fly Optimization Algorithm in the multivariable process fault diagnosis model, at the same time, to analyze the out of control sample data in the automobile crankshaft production. Compared with the neural network model in dealing with the fault diagnosis in multivariate process, Fruit Fly Optimization Algorithm's effectiveness s verified.
\end{abstract}

Keywords: Multivariate production process; Fruit Fly Optimization Algorithm; Fault diagnosis; BP artificial neural network

\section{Introduction}

In statistical process control (SPC), univariate techniques are designed to control the quality of the product by analyzing only one product characteristic. In most industrial production processes, however, there are several interrelated characteristics which jointly influence the quality of the final products. To avoid the over-control or undercontrol caused by univariate techniques, it is necessary to monitor the interrelated characteristics jointly and diagnose the quality failures to improve the consistency of process capability and product quality [1]. For multivariate production process quality control, through failure mode diagnosis, the abnormal variation of the product production characteristics can be discovered timely, the reason for the production process out of control can be found, then the corresponding measures can be taken to eliminate the abnormal variation to make the production system reach the stable status, thus the production rate can be improved and the reject ratio can be decreased [2]. In general, the failure mode diagnosis methods for multivariate production process quality control mainly include traditional statistics based methods and intelligent methods. While the intelligent failure mode diagnosis methods have been the popular methods nowadays [3].

The intelligent failure mode diagnosis methods can be classified from principle into five categories [4]: expert system based methods, model based methods, machine learning methods, artificial neural network (ANN) based methods and fuzzy-logic based methods. According to the current situation, the ANN is widely used due to its good prosperities such 
as parallelism, association memory, self-organization and self-learning etc. Czeslaw $\mathrm{T}$ Kowalski and Teresa Orlowska Kowalska applied two different ANN models to different failure diagnosis, the feed-forward neural network was used to identify all the failures and the Kohonen self-organization network was used to classify the failures [4]. Zheng Fatai et al. applied the BP neural network to failure diagnosis of hydraulic systems in construction machinery, through system data collecting, feature vector extracting and classifier constructing, based on the trained network, the learning algorithm was established and experiments showed that the neural network could give satisfactory results [5]. Huaqing Wang and Peng Chen presented an intelligent failure diagnosis method for rolling bearing based on probability theory and fuzzy neural network to correctly discover the correlation ship between the symptoms and failure modes [6].

However, in practice, some scholars found that the neural network has some deficiencies. Zhang Xinhai et al. thought that the BP ANN of slower convergence rate, studying and memory are of instability while applying the ANN to diagnose the mechanical failures [7]. Jian-bo $\mathrm{Yu}$ et al. applied the selectable neural network algorithm to multivariate production process failure mode diagnosis; they found that the algorithm is ease to fall into local optimal point easily [8]. Feng Huizong et al. thought that the traditional BP ANN has defects of long training time, slow convergence speed and low precision while it was used for failure diagnosis, thus they presented a new algorithm with combination of Particle Swarm Optimization (PSO) and BP ANN to train the weights and threshold of the neural network [9].

The deficiencies of the traditional neural network affect the effects of neural network based failure diagnosis in certain degree, in this paper, considering the advantages of the Fruit Fly Optimization Algorithm (FOA), a FOA based multivariate production process failure mode diagnosis model is given, then the presented algorithm is applied to automobile crankshaft production, and comparison with BP model is carried out to verify the effectiveness of the FOA.

\section{Research Methodology}

FOA was put forward by Dr. Pan Wenchao of Taiwan in 2011 who was inspired by foraging behavior of the fruit-flies. The algorithm is a new method which can search globally optimal solution and was deduced from foraging behavior of the fruit-flies. As the fruit-fly has developed olfactory organ and can better find various smells suspended in air, while approaching the food resource, it can quickly find the food and companions gathering location due to its keen sense of sight and then flies toward the direction [11]. The principle of FOA can be described as following [12].

(1) Give the initial position of a fruit-fly group.

(2) Give the random direction and distance of a single fruit-fly searching foods.

(3) As the position of the foods is unknown, Estimate the distance between the food and original point due to the unknown foods position, and calculate the decision value of taste concentration which is reciprocal value of the distance.

(4) Put the decision value of taste concentration into the decision function to obtain the taste concentration of the single fruit-fly's position.

(5) Find the fruit-fly of highest taste concentration.

(6) Contain the best taste concentration value and the corresponding ( $\mathrm{x}, \mathrm{y})$ coordination, then the fruit-fly group should fly toward the position.

(7) Execute step (2) (5) repetitively for iterative optimization, judge whether the current taste concentration is superior that of the previous iteration or not, if it is true then execute the step (6). 
The fruit-fly optimization algorithm is easy to be realized, it has strong global search ability and better accuracy and can be used for solving the extreme values of mathematical functions, coefficients optimization of Z-SCORE model, parameters optimization of general regression neural network, parameters optimization of gray neural network and parameters optimization of support vector machine, etc. [13]. The present study in this paper is belong to an application of general regression neural network parameters optimization.

\section{Presented Multivariate Production Process Failure Diagnosis Algorithm}

According to the FOA principle stated above and the characteristics of the multivariate production process failure diagnosis, the designed FOA based failure diagnosis algorithm flowchart is shown in Figure 1, the corresponding realization steps are as following.

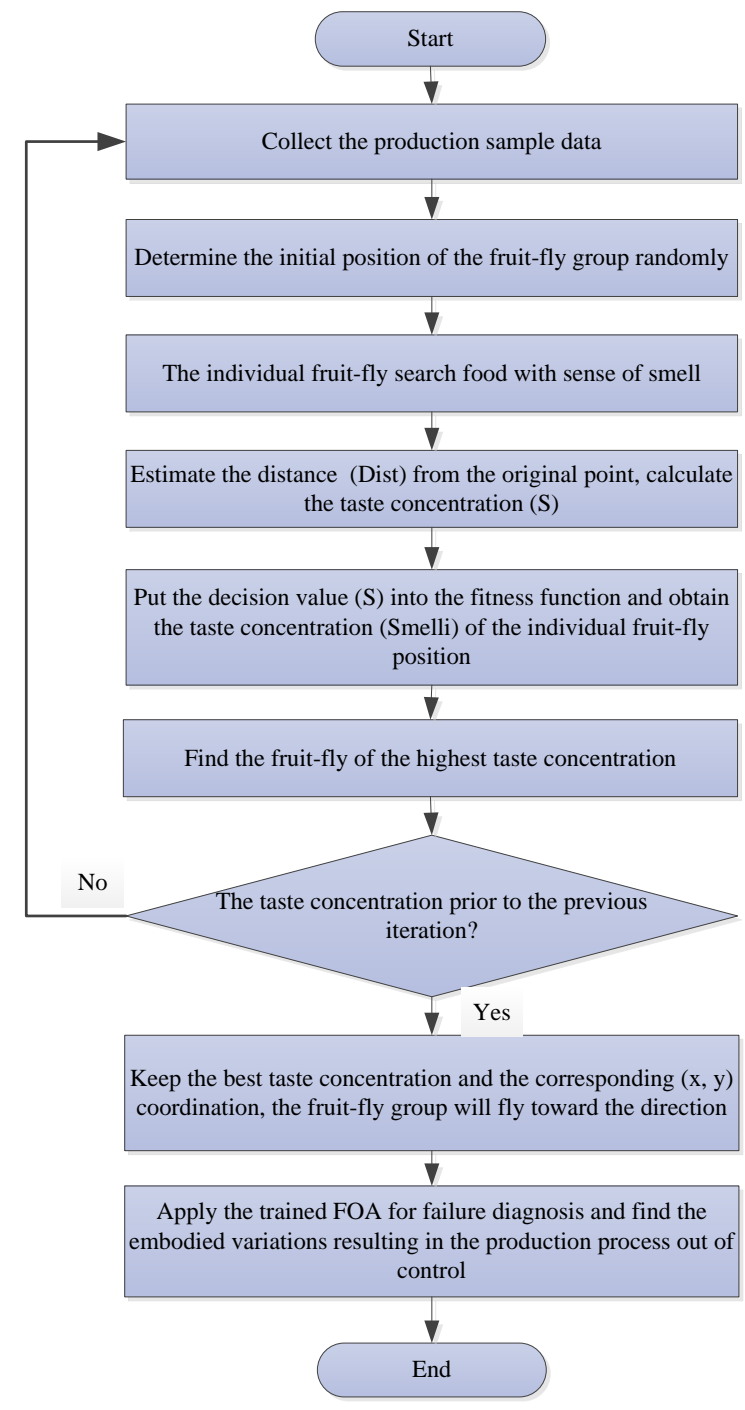

Figure 1. Flowchart of the Fruit-fly Optimization Algorithm

(1) Chose the initial position of the fruit-fly group randomly.

Init X_axis, Init Y_axis 
(2) The fruit-fly searches the distance and direction of the food randomly with its own sense of smell.

$X_{i}=\mathrm{X} \_$axis + Random Value, $Y_{i}=\mathrm{Y} \_$axis + Random Value

(3) Calculate the distance (Dist) between the found food and the original point and determine the taste concentration decision value (S).

$$
\text { Disti }=\sqrt{X_{i}^{2}+Y_{i}^{2}}, S_{i}=1 / \text { Disti }
$$

(4) Put $S$ into the fitness function and calculate the taste concentration of the individual fruit-fly (Smelli).

\section{Smelli $=$ Function $\left(S_{i}\right)$}

(5) Find the individual fruit-fly which has the maximum taste concentration.

$$
\text { [bestSmell bestIndex] }=\max (\text { Smell })
$$

(6) Keep the individual fruit-fly which has maximum taste concentration and the corresponding $(\mathrm{x}, \mathrm{y})$ coordination, the group will move toward the direction.

$$
\text { Smellbest }=\text { bestSmell }, X_{-} \text {axis }=X(\text { bestIndex }), Y_{-} \text {axis }=Y(\text { bestIndex })
$$

(7) Execute the step (2) step (5) and judge the taste concentration, if the calculated current value is prior to the previous, then execute step (6). From the flow, the detailed position of the food which has the best taste concentration.

(8) Applying the trained FOA for failure diagnosis can find the variables or variable set resulting in the process out-of-control.

\section{Case Application}

In an automobile crankshaft production factory, considering the production characteristics, the main bearing journal diameter $X_{1}$, rod journal diameter $X_{2}$ and stroke $X_{3}$ of crankshaft shaped by the main bearing journal and rod journal are selected as variables, MATLAB R2011a software is used to generate enough training sample data of various abnormal patterns with $2 \sigma$ offset.

The mean value $\left(X_{1}, X_{2}, X_{3}\right)$ of the training sample data can be determined as following. :

$(89.96+2 * 0.0313,73.46,67.40)$

$(89.96,73.46+2 * 0.2739,67.40)$,

$(89.96,73.46,67.40+2 * 0.2706)$,

$(89.96+2 * 0.0313,73.46+2 * 0.2739,67.40)$,

$(89.96+2 * 0.0313,73.46,67.40+2 * 0.2706)$,

$(89.96,73.46+2 * 0.2739,67.40+2 * 0.2706)$,

$(89.96+2 * 0.0313,73.46+2 * 0.2739,67.40+2 * 0.2706)$.

The covariance matrix is :

$$
\Sigma=\left[\begin{array}{ccc}
0.0010 & -0.0020 & 0.0008 \\
-0.0020 & 0.0750 & -0.0085 \\
0.0008 & -0.0085 & 0.0732
\end{array}\right]
$$

The training sample data include all abnormal patterns, 350 groups of data are generated, the principle component analysis is used to decrease the dimensions of data and only two principle components are contained. Thus, the input of the design FOA is a 2-dimension matrix $\left(X_{1}^{\prime}, X_{2}^{\prime}\right)$ and the output is a 3-dimensional matrix.

To verify the effectiveness of the presented FOA, a BP-ANN model is built and the comparison of the two methods is carried out. 


\subsection{Applying FOA for Failure Mode Diagnosis}

(1) Determining the input and output

Aiming at the study object, the output variable is set as $T=\left(T_{1}, T_{2}, T_{3}\right)$, where $T_{i}$ represents the state of the $i^{\text {th }}$ variable, $T_{i}=1$ shows that the variable $X_{i}$ is out of control, while $T_{i}=0$ shows that the variable is under control. Thus in this case, there are seven notable output patterns, as is shown in the Table 1.

Table 1. Output Patterns of the FOA

\begin{tabular}{|c|c|c|}
\hline Variable(s) out of control & Variable(s) under control & Input \\
\hline$T_{1}$ & $T_{2}$ and $T_{3}$ & $T=(1,0,0)^{T}$ \\
\hline$T_{2}$ & $T_{1}$ and $T_{3}$ & $T=(0,1,0)^{T}$ \\
\hline$T_{3}$ & $T_{1}$ and $T_{2}$ & $T=(0,0,1)^{T}$ \\
\hline$T_{1}$ and $T_{2}$ & $T_{3}$ & $T=(1,1,0)^{T}$ \\
\hline$T_{1}$ and $T_{3}$ & $T_{2}$ & $T=(1,0,1)^{T}$ \\
\hline$T_{2}$ and $T_{3}$ & $T_{1}$ & $T=(0,1,1)^{T}$ \\
\hline$T_{1}, T_{2}$ and $T_{2}$ & - & $T=(1,1,1)^{T}$ \\
\hline
\end{tabular}

In general, when there are $p$ variables, the number of the failure modes is $2^{\mathrm{p}}-1$.

(2) Determining the group scale and number of iterations

The number of iterations is determined according to the specific training effect for the designed FOA in this study, through multiple tests, the number is set as 1000 . The randomly selected initial position interval of the fruit-fly group is $[0,1]$, the flying distance interval of fruit-fly searching food is $[-10,10]$.

(3) Training

While the relevant parameters of FOA are set, training can be carried out. It should be noticed that the selected sample data should be of integrity, representativeness and representativeness, the aim of training is to find out the mapping relationship between the input and output of the sample data, thus multiple attempts are needed to select right training time.

\subsection{Applying BP Neural Network BP for Failure Mode Diagnosis}

For the input and output of the BP-ANN, the number of the output layer neurons is equal to the number of variables. As is same to FOA, the number of the failure modes is $2^{p}-1$, the detailed output pattern classifying is shown in the Tab.1.

While design the structure of a BP-ANN, layer number, neurons of each layer, expected error, learning rate, momentum factor and training function etc. should be taken into account.

1) For the layer number, in general firstly a hidden layer is considered, if augmenting node of hidden layer cannot satisfy the network precision, augmenting number of hidden layer is considered.

2) For nodes of hidden layer, the selecting principle is that in the premise of satisfying the precision requirement and resolving the problem, through comparing of training different nodes and a little allowance is considered, then the network is determined for failure pattern diagnosis. 
3) For the expected error, in general, two networks with different expected error can be trained simultaneously; the suitable network can be selected considering the relative factors comprehensively.

4) For learning ratio, the learning ratio determines the variances of weighs in training. In general, smaller learning ratio can ensure the system stability and the optimal selection scope is [0.01-0.8]. Meanwhile, to overcome the defects of getting into local minimum and slow convergence rate in BP neural network, the momentum factor should be added to the weigh variation, the factor is usually a constant selected in $(0,1)$.

Considering the parameter selecting experience above, for the designed BP neural network in this study, the number of the training sample is 350 . The network consists of input layer, a hidden layer and output layer, and the unit number of each layer is 2, 15 and 3 individually. The expected error is 0.000001 , the learning ratio is 0.01 and the momentum is 0.9 , the cycling time of the network is 1000 .

After the parameters are determined, the network can be trained: the collected data are divided into training samples and texting samples, the training samples are used for network training and the testing samples are used for testing the network generalization.

Through multiple training attempts, the training functions of each layer are tansig function, tansig function and logsig function individually.

\subsection{Diagnosis Results and Comparison}

In testing stage, enough out-of-control sample data of 7 different abnormal patterns are generated with MATLAB R2011a, there are 20 sets of out-of-control sample data with every different abnormal pattern, thus total 140 groups of data are obtained, 40 of them are shown in Table 2.

Table 2. Testing Sample Data

\begin{tabular}{|c|c|c|c|c|c|c|c|}
\hline $\begin{array}{l}\text { Serial } \\
\text { number }\end{array}$ & $X_{1} / \mathrm{mm}$ & $X_{2} / \mathrm{mm}$ & $X_{3} / \mathrm{mm}$ & $\begin{array}{l}\text { Serial Variable } \\
\text { number }\end{array}$ & $X_{1} / \mathrm{mm}$ & $X_{2} / \mathrm{mm}$ & $X_{3} / \mathrm{mm}$ \\
\hline 1 & 90.093 & 73.501 & 67.401 & 21 & 89.960 & 74.467 & 68.024 \\
\hline 2 & 90.100 & 73.404 & 67.305 & 22 & 90.068 & 74.013 & 67.506 \\
\hline 3 & 90.061 & 73.751 & 66.683 & 23 & 89.994 & 74.535 & 67.790 \\
\hline 4 & 90.090 & 73.272 & 68.241 & 24 & 89.965 & 74.766 & 66.835 \\
\hline 5 & 90.096 & 73.458 & 67.544 & 25 & 89.877 & 74.774 & 67.282 \\
\hline 6 & 90.087 & 73.999 & 67.438 & 26 & 89.957 & 74.738 & 67.046 \\
\hline 7 & 90.093 & 73.667 & 67.372 & 27 & 89.982 & 74.783 & 67.038 \\
\hline 8 & 90.102 & 73.200 & 67.647 & 28 & 89.968 & 74.409 & 68.186 \\
\hline 9 & 90.073 & 73.654 & 66.719 & 29 & 89.991 & 74.515 & 67.596 \\
\hline 10 & 90.120 & 73.470 & 67.257 & 30 & 90.006 & 74.406 & 66.910 \\
\hline 11 & 90.056 & 74.173 & 66.970 & 31 & 89.953 & 74.717 & 67.147 \\
\hline 12 & 90.064 & 74.003 & 67.038 & 32 & 90.024 & 74.423 & 67.667 \\
\hline 13 & 90.114 & 73.275 & 67.681 & 33 & 90.019 & 74.623 & 66.724 \\
\hline 14 & 90.091 & 73.980 & 67.211 & 34 & 89.924 & 74.727 & 66.967 \\
\hline 15 & 90.093 & 72.848 & 67.836 & 35 & 89.964 & 74.811 & 66.896 \\
\hline 16 & 90.106 & 73.260 & 67.518 & 36 & 89.941 & 74.774 & 67.456 \\
\hline 17 & 90.128 & 73.437 & 67.869 & 37 & 90.002 & 74.529 & 67.046 \\
\hline 18 & 90.084 & 73.643 & 67.067 & 38 & 89.957 & 74.666 & 67.866 \\
\hline 19 & 90.105 & 73.351 & 67.330 & 39 & 89.938 & 74.613 & 67.772 \\
\hline 20 & 90.088 & 73.516 & 67.741 & 40 & 89.953 & 74.565 & 66.731 \\
\hline
\end{tabular}


The principle component analysis (PCA) is used to decrease the dimensions of sample data, the processed data are taken as the input to train the designed FOA and BP-ANN, and the trained FOA and BP-ANN are used for failure pattern diagnosis, the results of training and failure pattern diagnosis are as following.

The training process of FOA is shown in Figure 2 and Figure 3. The training of BP-ANN is shown in Figure 4. The diagnosis results of FOA and BP-ANN are listed in Table 3 and Table 4 respectively.

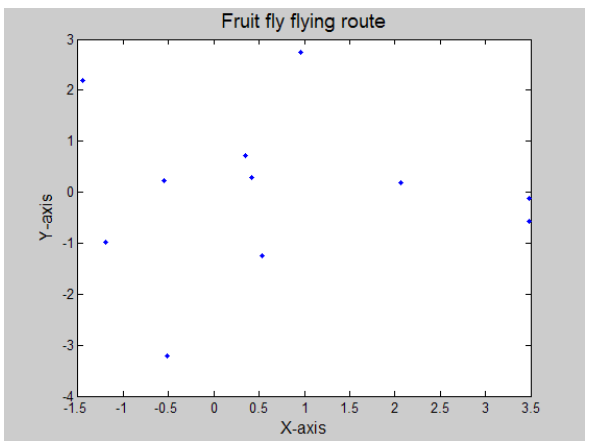

Figure 2. Track of the FOA

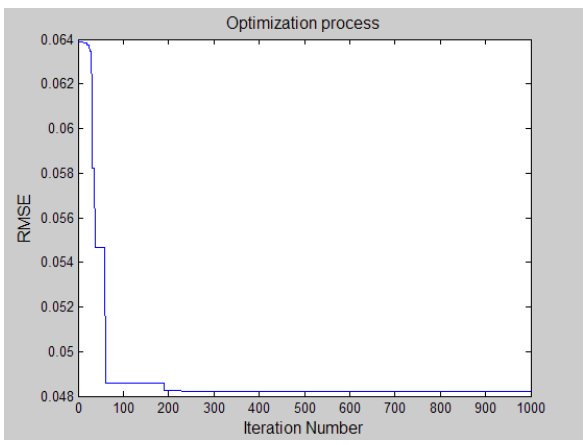

Figure 3. Training of FOA

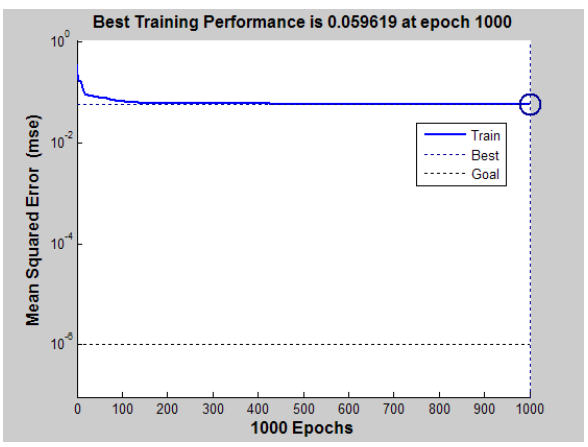

Figure 4. Training of BP-ANN

Table 3. FOA Diagnosis Results

\begin{tabular}{|c|c|c|c|c|c|c|c|}
\hline Diagnosis pattern & $(1,0,0)$ & $(0,1,0)$ & $(0,0,1$ & $(1,1,0)$ & $(1,0,1)$ & $(0,1,1)$ & $(1,1,1)$ \\
\hline Diagnosis number & 19 & 23 & 22 & 19 & 18 & 20 & 19 \\
\hline
\end{tabular}


Table 4. BP-ANN Diagnosis Results

\begin{tabular}{|c|c|c|c|c|c|c|c|}
\hline Diagnosis pattern & $(1,0,0)$ & $(0,1,0)$ & $(0,0,1$ & $(1,1,0)$ & $(1,0,1)$ & $(0,1,1)$ & $(1,1,1)$ \\
\hline Diagnosis number & 25 & 14 & 28 & 15 & 29 & 15 & 14 \\
\hline
\end{tabular}

From the training results, the diagnosis effects al can meet the requirements after the two models are trained. The FOA can meet the demands of given precision after 230 iterations while the BP algorithm needs about 420 iterations, thus the FOA is of higher efficiency. In addition, from the FOA building procedure, it can be seen that fewer parameters are required to be set artificially in advance than those of BP algorithm, then it is easy to operate. Also, from the diagnosis effect, the FOA is of higher accuracy than BP neural network model.

So, it can be concluded that from the example, the FOA is obviously more advantageous than BP neural network model in multivariate failure mode diagnosis of automobile crankshaft production.

\section{Concluding Remarks and Future Research Directions}

In this work, the principle and characteristics of FOA is analyzed and a FOA based failure mode diagnosis method for multivariate production quality control is presented. Taking the automobile crankshaft production process as an example, the corresponding FOA and BP neural network model are built individually. From the comparison between the training procedures of failure mode diagnosis, FOA has shorter training times than the BP neural network model and the bad effect of uncertainty owing to setting necessary network structure parameters in BP model building on the failure mode diagnosis result can be avoided. So it is of good application prospect in multivariate production quality control. While it should be noted that this article focuses on the feasibility of applying FOA in multivariate production quality control, other algorithm characteristics such as the complexity and efficiency etc. of the FOA should be studied in future work.

\section{Acknowledgements}

This research is supported by Nature Science Foundation of China (Grant No. 61402361, Grant No. 60903124), Research and Development Program of Shaanxi Province for Young Star in Science \& Technology (Grant No. 2012KJXX-34), Scientific Research Plan of Xi'an University of Technology, China (Grant No. 102-211305) and Science \& Technology Project of Shaanxi Province, China [14JK1521].

\section{References}

[1] S. Barceló, S. Vidal-Puig and A. Ferrer, "Quality and Reliability Engineering International”, vol. 1, no. 27, (2010).

[2] G. Stefatos and A. B. Hamza, "Expert Systems with Applications, vol. 3, no. 36, (2009).

[3] S. Li, "China Mechanical Engineering", vol. s1, no. 17, (2006).

[4] B. Zhang, Y. Xu and Q. Pan, "Aircraft Design", vol. 5, no. 27, (2007).

[5] C. T. Kowalski and T. Orlowska-Kowalska, "Mathematics and Computers in Simulation", vol. 3, no. 63, (2003).

[6] F. Zheng, J. Ye and Z. Wenf, "Construction Machinery and Equipment”, vol. 10, no. 37, (2006).

[7] H. Wang and P. Chen, "Computers \& Industrial Engineering", vol. 4, no. 60, (2011).

[8] X. Zhang and Y. Lei, "Noise and Vibration Control", vol. 5, no. 28, (2008).

[9] J.-b. Yu and L.-f. Xi, "Expert Systems with Applications", vol. 1, no. 3, (2009).

[10] H.-z. Feng, X.-m. Wu, R.-d. Yuan and L. Su, "Control and Instruments in Chemical Industry", vol. 1, no. 40, (2013).

[11] W.-T. Pan, "Knowledge-Based Systems", vol. 2, no. 26, (2012) 
[12] D. Shi, J. Lu and L. Lu, "Journal of Wuhan Unicersity of Technology", vol. 3, no. 34, (2012).

[13] H. Li, G. Sen and C. Li, “A Journal of Quantitatice Economics”, vol. 3, no. 29, (2012).

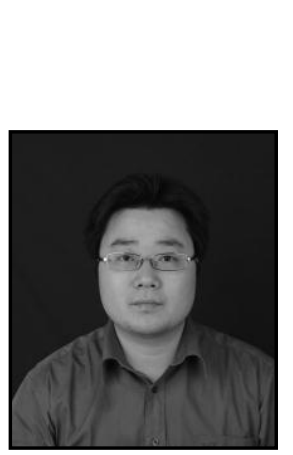

\begin{abstract}
Authors
Y. Mingshun, He received the B.S. degree and M.S. degree from Yanshan University, Qinhuangdao, China in 1995 and 1998 respectively. He received the $\mathrm{PhD}$. degree in Mechanical Engineering from Xi'an Jiaotong University, Xi'an, China. He is currently an associate professor in the School of Mechanical and Precision Instrument Engineering, Xi'an University of Technology, Xi'an, China. He has authored / coauthored over 30 publications and two book chapters. His research interests include production system designing and planning, integrated quality management and advanced manufacturing technology.
\end{abstract}

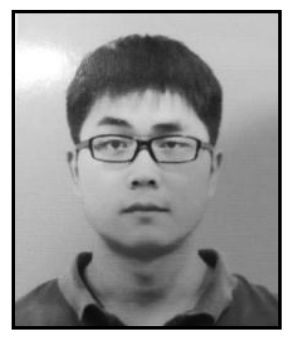

Kong Xiangjian, He is a M.S. student in School of Mechanical and Precision Instrument Engineering, Xi'an University of Technology (XAUT), Xi'an, China. He received his B.S. degree in Industrial Engineering in 2013 from Henan University of Science and Technology. His research interests include reliability and quality control, Nanoindentation and advanced manufacturing technology.

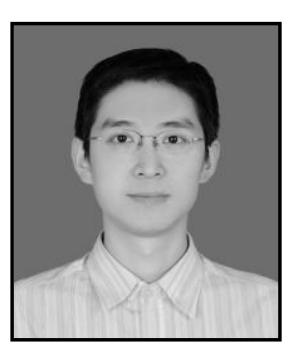

Liu Yong, He received the M.S. degree and PhD. degree from Xi' an University of Technology (XAUT), Xi'an, China in 2006 and 2009 respectively. He is currently an associate professor in School of Mechanical and Precision Instrument Engineering, XAUT. He has authored/coauthored over 20 publications. His research interests include production system designing and advanced manufacturing technology.

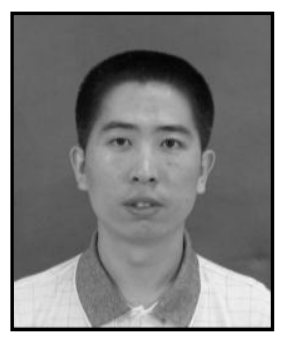

Gao Xinqin, He received his BS degree in Mechanical and Electrical Engineering from Northwest Institute of Light Industry in 1999, his MS degree in Manufacturing Engineering and Automation from Shaanxi University of Science and Technology in 2004, and his $\mathrm{PhD}$ degree in Mechanical Engineering from Xi'an Jiaotong University in 2008. He is currently an Associate Professor in the School of Mechanical and Precision Instrument Engineering, XAUT .He has authored/coauthored over 30 publications and a book. His research interests include manufacturing systems modeling, workflow management technology, business process reengineering, product conceptual design, enterprise information integration and polychromatic sets theory. 


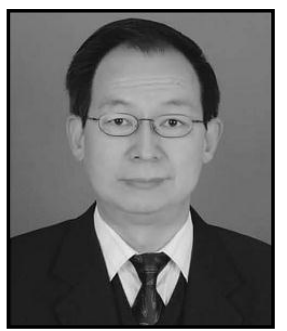

Li Yan, He received the B.S. degree and M.S. degree from Xi'an University of Technology (XAUT), Xi'an, China in 1983 and 1986 respectively. He received the Ph.D. degree in Aeronautics and Astronautics Manufacturing Engineering from Northwest Polytechnical University (NWPU), Xi'an, China in 1995. He is currently a professor in the School of Mechanical and Precision Instrument Engineering, XAUT. He is currently the vice-president of XAUT. He has authored/coauthored over 100 publications and four book chapters. His research interests include advanced precision plastic forming technology, production planning and control; difficult-to-process material machining and control, process planning optimization. 